\title{
ON DOMINATION TOPOLOGICAL INDICES OF GRAPHS
}

\author{
A.M. HANAN AHMED ${ }^{1, *}$, ANWAR ALWARDI ${ }^{2}$, M. RUBY SALESTINA ${ }^{1}$ \\ ${ }^{1}$ Department of Mathematics, Yuvaraja's college, University of Mysore, Mysuru, India \\ ${ }^{2}$ Department of Mathematics, University of Aden, Yemen
}

*Corresponding author: hananahmed1a@gmail.com

\begin{abstract}
Topological indices and domination in graphs are the essential topics in the theory of graphs. A set of vertices $D \subseteq V(G)$ is said to be a dominating set for $G$ if any vertex $v \in V-D$ is adjacent to some vertex $u \in D$. In this research work, we define a new degree of each vertex $v \in V(G)$, called the domination degree of $v$ and denoted by $d_{d}(v)$, along with this new degree some domination indices based on domination degree are introduced. We study some basic properties of the domination degree function. Exact values and bounds for domination Zagreb indices of some families of graphs including the join and corona product are obtained. Finally, we generalize the domination degree of the vertex and new general indices are defined.
\end{abstract}

\section{INTRODUCTION}

In this research article, we assume that $G=(V, E)$ is a connected simple graph. In the field of chemistry, graph theory has provided many useful tools, such as topological indices. Chem-informatics is one of the latest concepts which is a join of chemistry, mathematics, and information science.

Topological indices are numerical parameters of the graph, such that these parameters are the same for the

Received September $21^{\text {st }}, 2020$; accepted October $20^{\text {th }}, 2020$; published November $24^{\text {th }}, 2020$.

2010 Mathematics Subject Classification. 05C69, 05C90, 05C35.

Key words and phrases. domination Zagreb indices; domination degree; minimal dominating set; total number of minimal domination sets.

(C)2021 Authors retain the copyrights of their papers, and all open access articles are distributed under the terms of the Creative Commons Attribution License. 
graph which they are isomorphism. Some of the major classes of topological indices are distance basedtopological indices (see [2], [3]) and degree- based-topological indices (see [5], [20], [23]). Degree basedtopological indices are of great significance. The Wiener index $W(G)$ is the old index and the first distancebased, introduced by chemist Wiener [24] in 1974. After the introduction of the Wiener index, many another distance-based topological indices, have been proposition and take into consideration in chemical and mathematical chemical literature. For example, Harary index [17] and eccentric connectivity index [22]. presently a great number of vertex-degree-based graph invariants are being studied in mathematical and mathematical chemical literature ( [8], [9]). Among them, the Zagreb indices $M_{1}(G)$ and $M_{2}(G)$ are the most widely investigated. Those have been inserted more than forty years ago ( [11], [12]), which are defined as follows:

$$
\begin{gathered}
M_{1}(G)=\sum_{v \in V(G)} d^{2}(v)=\sum_{u v \in E(G)} d(u)+d(v) \\
M_{2}(G)=\sum_{u v \in E(G)} d(u) d(v) .
\end{gathered}
$$

For properties of the two Zagreb indices see [10] and the papers cited therein. The Zagreb co-indices defined in [21], and are given by :

$\bar{M}_{1}(G)=\sum d(u)+d(v)$ and $\quad \bar{M}_{2}(G)=\sum d(u) d(v)$, where $u v$ is not an edge in $E(G)$. The degree of a vertex $u$ in $G, d(u)$ is the number of edges that are incident to $u$ in $G$. The maximum and minimum degrees of vertices of a graph $G$ are denoted by $\Delta(G)$ and $\delta(G)$ respectively. $\bar{G}$ is the complement of a graph $G$, having the same vertex set of $G$ so that two vertices of $\bar{G}$ are neighboring if and only if they are not neighboring in $G$. If for every two vertices $u, v \in V$, there exists a $(u, v)$-path in $G$, then $G$ is connected, otherwise, $G$ is said to be disconnected. A set $D \subseteq V$ is said to be a dominating set of $\mathrm{G}$, if for any vertex $v \in V-D$ there exists a vertex $u \in D$ such that $u$ and $v$ are adjacent. The domination number $\gamma(G)$ of $\mathrm{G}$ is the minimum cardinality of a minimal dominating set in $\mathrm{G}$. The upper domination number $\Gamma(G)$ of $\mathrm{G}$ is the maximum cardinality of a minimal dominating set in G [4]. For a survey of domination in graphs, refer to ( [14], [15]). A dominating-set $D=\left\{v_{1}, v_{2}, \ldots, v_{r}\right\}$ is minimal if $D-v_{i}$ is not a dominating set. We use $T_{m}$ to denote the number of minimal dominating sets. In [7] a graph has at most $O\left(1.7159^{n}\right)$ minimal dominating-sets and there exist graphs with at least $O\left(1.5705^{n}\right)$ minimal dominating-sets. For more definitions or properties, we refer to ([7], [18], [19]).

\section{Domination Degree in Graphs}

In this partition, we sitting the definition of domination degree of the vertex $v$. We consider the lower and upper bound for this degree, and we study some basic properties of the domination degree.

\section{Definition 2.1.}


For each vertex $v \in V(G)$, the domination degree denoted by $d_{d}(v)$ and defined as the number of minimal dominating sets of $G$ which contains $v$.

The minimum domination and maximum domination degree of $G$ are denoted by $\delta_{d}(G)=\delta_{d}$ and $\Delta_{d}(G)=$ $\Delta_{d}$ respectively, where

$\delta_{d}=\min \left\{d_{d}(v): v \in V(G)\right\}$ and $\Delta_{d}=\max \left\{d_{d}(v): v \in V(G)\right\}$.

Let $v \in V(G)$ and $v^{\prime} \in G^{\prime}$. Then $d_{d G}(v)=d_{d G^{\prime}}\left(v^{\prime}\right)$ if $G \cong G^{\prime}$.

The domination degree function is obviously invariant under isomorphism.

\section{Observation 2.1.}

$1 \leq d_{d}(v) \leq T_{m}(G)$, where $T_{m}(G)$ denotes the total number of minimal dominating sets.

\section{Observation 2.2.}

Suppose $G$ is a graph of $n \geq 2$ vertices having $\Delta(G)=n-1$. Then $\gamma(G)=1$ and $d_{d}(v) \geq 1$, for any $v \in V(G)$. Also, $d_{d}(v)=1$ if and only if $d(v)=n-1$.

\section{Corollary 2.1.}

If $G \cong K_{n}$ and $\bar{G}$ is the complement of $G$, then

$d_{d G}(v)=d_{d \bar{G}}(v)$, and $T_{m}(\bar{G})=1$.

\section{Observation 2.3.}

Let $G(V, E)$ be a graph with minimal dominating sets $S_{1}, S_{2}, \ldots, S_{t}$. Then

$t \gamma(G) \leq \sum_{v \in V(G)} d_{d}(v) \leq t \Gamma(G)$.

We use the notion $\rho(G)=\sum_{v \in V(G)} d_{d}(v)$.

\section{Proposition 2.1.}

Let $G$ be the complete bipartite graph $K_{r, s}$. Then $d_{d \bar{G}}(v)=d_{G}(v)$.

Proof. Let the bipartite sets of $K_{r, s}$ be $A$ and $B$, where $A$ contains the vertices of degree $s$ and $B$ contains the vertices of degree $r$. Also $T_{m}(\bar{G})=r s$, such that if $v \in A \Rightarrow d_{d \bar{G}}(v)=s$. similarly if $v \in B \Rightarrow$ $d_{d \bar{G}}(v)=r$.

\section{Observation 2.4.}

Let $G=\bigcup_{i=1}^{t} G_{i}$ be the disjoint union of graphs $G_{1}, G_{2}, \ldots, G_{t}$. Then $\gamma(G)=\sum_{i=1}^{t} \gamma\left(G_{i}\right)$ and $T_{m}(G)=$ $\prod_{i=1}^{t} T_{m}\left(G_{i}\right)$. For $v \in V\left(G_{i}\right), d_{d G}(v)=d_{d G_{i}}(v) \prod_{j=1}^{t} T_{m}\left(G_{j}\right), j \neq i$.

\section{Proposition 2.2.}

Given $H$ is a spanning subgraph of $G$ with $V(H)$ is the same as $V(G)$. If the domination number of $H$ is the same as the domination number of $G$, then $T_{m}(H) \leq T_{m}(G)$. 
Proof. Use the first presumption, every dominating set for $H$ is also a dominating set of $G$. As $\gamma(H)=\gamma(G)$, its ensured that every minimal dominating set of minimum cardinality for $H$ is also a minimal dominating set of minimum cardinality for $G$.

\section{Definition 2.2.}

The graph $G$ is called $k$-domination regular graph if and only if $d_{d}(v)=k$ for all $v \in V(G)$.

Example 2.1. $S_{r}$ and $K_{n}$ are 1 -domination regular graph.

\section{Proposition 2.3.}

Let $G$ be the double star graph $S_{r, s}$. Then

$T_{m}(G)=4$, and any double star graph is 2 -domination regular graph.

Proof. Let $\left\{v, v_{1}, \ldots, v_{r-1}, w, w_{1}, \ldots, w_{s-1}\right\}$ be the set of all vertices of $G$ with $\{v, w\}$ be the center vertices. There are four type of minimal dominating sets as following: $\{v, w\},\left\{v_{1}, v_{2}, \ldots, v_{r-1}, w_{1}, w_{2}, \ldots, w_{s-1}\right\}$, $\left\{v, w_{1}, w_{2}, \ldots, w_{s-1}\right\}$ and $\left\{w, v_{1}, v_{2}, \ldots, v_{r-1}\right\}$.

$\Rightarrow T_{m}(G)=4$. From this we get, $d_{d}(v)=2$ for all $v \in V(G)$.

\section{Domination Zagreb Indices of a Graph}

\section{Definition 3.1.}

Let $G$ be a simple connected graph, the first domination, second domination Zagreb and modified first Zagreb indices are define as :

$$
\begin{gathered}
D M_{1}(G)=\sum_{v \in V(G)} d_{d}^{2}(v), \\
D M_{2}(G)=\sum_{u v \in E(G)} d_{d}(u) d_{d}(v), \\
D M_{1}^{*}=\sum_{u v \in E(G)}\left[d_{d}(u)+d_{d}(v)\right] .
\end{gathered}
$$

\section{Lemma 3.1.}

$T_{m}\left(S_{r}\right)=2$ and $T_{m}\left(K_{n}\right)=n$.

For all $v \in V\left(S_{r}\right)$ or $v \in V\left(K_{n}\right)$, we get $d_{d}(v)=1$.

\section{Proposition 3.1.}

(1) For the star graph $S_{r}$, with $r+1$ vertices.

$$
D M_{1}\left(S_{r}\right)=r+1, \quad D M_{2}\left(S_{r}\right)=r \quad \text { and } \quad D M_{1}^{*}\left(S_{r}\right)=2 r .
$$

(2) For the complete graph $K_{n}$

$$
D M_{1}\left(K_{n}\right)=n \quad, D M_{2}\left(K_{n}\right)=\frac{n(n-1)}{2} \text { and } D M_{1}^{*}\left(K_{n}\right)=n(n-1) .
$$


(3) For the double star graph $S_{r, s}$

$$
D M_{1}\left(S_{r, s}\right)=4(r+s+2) \quad D M_{2}\left(S_{r, s}\right)=4(r+s+1) \quad \text { and } \quad D M_{1}^{*}\left(S_{r, s}\right)=4(r+s+1) \text {. }
$$

\section{Lemma 3.2.}

$T_{m}\left(K_{r, s}\right)=r s+2$ and $d_{d}(v)=\left\{\begin{array}{c}r+1 ; \\ s+1\end{array}\right.$

for all $v \in V\left(K_{r, s}\right)$.

\section{Theorem 3.1.}

If $G \cong K_{r, s}$, then

$$
\begin{gathered}
D M_{1}(G)=r(r+1)^{2}+s(s+1)^{2}, \\
D M_{2}(G)=(r+1)(s+1) r s, \\
D M_{1}^{*}(G)=r s(r+s+2) .
\end{gathered}
$$

Proof. By using the definition of domination Zagreb indices and lemma 3.2, we get the results.

\section{Corollary 3.1.}

Let $G$ be the complete bipartite $K_{r, s}$. Then

(1) $D M_{1}(G)=M_{1}(G)+4 r s+(r+s)$.

(2) $D M_{2}(G)=M_{2}(G)+M_{1}(G)+r s$.

(3) $D M_{1}^{*}(G)=M_{1}(G)+2 r s$.

Proof. In the complete bipartite $K_{r, s}$, we can see that $d_{d}\left(v_{i}\right)=d\left(v_{i}\right)+1$ for all $i=1,2, \ldots, r+s$

$$
\begin{aligned}
D M_{1}(G) & =\sum_{v \in V(G)} d_{d}^{2}(v)=\sum_{v \in V(G)}(d(v)+1)^{2} \\
& =M_{1}(G)+4 r s+(r+s)
\end{aligned}
$$

And,

$$
\begin{aligned}
D M_{2}(G) & =\sum_{u v \in E(G)} d_{d}(u) d_{d}(v)=\sum_{u v \in E(G)}(d(u)+1)(d(v)+1) \\
& =M_{2}(G)+M_{1}(G)+r s \\
D M_{1}^{*}(G) & =\sum_{u v \in E(G)}\left[d_{d}(u)+d_{d}(v)\right]=\sum_{u v \in E(G)}[(d(u)+1)+(d(v)+1)] \\
& =M_{1}(G)+2 r s .
\end{aligned}
$$

\section{Proposition 3.2.}


If $G \cong K_{r, s}$, then

(1) $D M_{1}(\bar{G})=D M_{1}^{*}(\bar{G})=M_{1}(G)$.

(2) $D M_{2}(\bar{G})=\overline{M_{2}}(G)$.

Proof. See proposition 2.1.

\section{Lemma 3.3.}

Let $G$ be the Windmill graph $W d_{r}^{s}$. Then $T_{m}\left(W d_{r}^{s}\right)=(r-1)^{s}+1$. And $d_{d}(v)= \begin{cases}1, & \text { if } v \text { is the center vertex } \\ (r-1)^{s-1}, & \text { otherwise. }\end{cases}$

\section{Theorem 3.2.}

If $G \cong W d_{r}^{s}$, then

$$
\begin{gathered}
D M_{1}(G)=1+s(r-1)^{2 s-1} \\
D M_{2}(G)=s\left((r-1)^{s}+(r-1)^{2 s-1}\left(\frac{r-2}{2}\right)\right), \\
D M_{1}^{*}(G)=s(r-1)\left(1+(r-1)^{s}\right) .
\end{gathered}
$$

Proof.

$$
\begin{aligned}
D M_{1}(G) & =\sum_{v \in V(G)} d_{d}^{2}(v)=1+\sum_{v \in V(G)-1} d_{d}^{2}(v) \\
& =1+(r-1)^{2(s-1)}(|V(G)|-1) \\
& =1+s(r-1)^{2 s-1} .
\end{aligned}
$$

Let $E_{1}$ denote the set of all edges which are incident with the center vertex and $E_{2}$ be the set of all edges of the complete graph, then

$$
\begin{aligned}
D M_{2}(G) & =\sum_{u v \in E(G)} d_{d}(u) d_{d}(v)=\sum_{u v \in E_{1}} d_{d}(u) d_{d}(v)+\sum_{u v \in E_{2}} d_{d}(u) d_{d}(v) \\
& =(r-1)^{s-1}\left|E_{1}\right|+s(r-1)^{2 s-2}\left|E_{2}\right| \\
& =s\left((r-1)^{s}+(r-1)^{2 s-1}\left(\frac{r-2}{2}\right)\right), \\
D M_{1}^{*}(G)= & \sum_{u v \in E(G)}\left[d_{d}(u)+d_{d}(v)\right]=\sum_{u v \in E_{1}}\left[d_{d}(d)+d_{d}(v)\right]+\sum_{u v \in E_{2}}\left[d_{d}(u)+d_{d}(v)\right] \\
= & \left(1+(r-1)^{s-1}\right)\left|E_{1}\right|+2 s(r-1)^{s-1}\left|E_{2}\right| \\
= & s(r-1)\left(1+(r-1)^{s}\right) .
\end{aligned}
$$




\section{Proposition 3.3.}

If $G$ is $K$-domination regular graph with $n$ vertices, and $m$ edges, then

$D M_{1}(G)=n K^{2}, \quad D M_{2}(G)=m K^{2} \quad$ and $\quad D M_{1}^{*}(G)=2 m K$.

\section{Definition 3.2.}

Let $P_{3}$ be the 3 vertex tree, is rooted in one of its terminal vertices. For $k=2,3,4, \ldots$ build the rooted tree $B_{k}$ by identifying the roots of $k$-copies of $P_{3}$. The vertex obtained by identifying the roots of $P_{3}-$ trees is the root of $B_{k}[16]$.

\section{Definition 3.3.}

Let $d \geq 2$ be an integer. Let $\beta_{1}, \beta_{2}, \ldots, \beta_{d}$ be as specified in Definition 3.2 i.e., $\beta_{1}, \beta_{2}, \ldots, \beta_{d} \in\left\{B_{2}, B_{3}, \ldots\right\}$. A Kragujevac tree $T$ is a tree has a vertex of degree $d$, neighboring to the roots of $\beta_{1}, \beta_{2}, \ldots, \beta_{d}$. This vertex be the central- vertex of $T$, where $d$ is the degree of the tree $T$. The subgraphs $\beta_{1}, \beta_{2}, \ldots, \beta_{d}$ are the branches of $T$. Recall that some (or all) branches of T may be mutually isomorphic [16].

The branch $B_{k}$ has $2 k+1$ vertices. Therefore, if in the Kragujevac tree T, specified in Definition 3.3, $\beta_{i} \cong \beta_{k_{i}}, i=1,2, \ldots, d$ then its order is $n(T)=1+\sum_{i=1}^{d}\left(2 k_{i}+1\right)$.

\section{Proposition 3.4.}

Let $T$ be the Kragujevac tree of order $n(T)=1+\sum_{i=1}^{d}\left(2 k_{i}+1\right)$ and size $m$. Then

$$
\begin{gathered}
D M_{1}(T)=4\left[1+\sum_{i=1}^{d}\left(2 k_{i}+1\right)\right], \\
D M_{2}(T)=D M_{1}^{*}(T)=4 m .
\end{gathered}
$$

Proof. It is easy to see that, in the Kragujevac tree of order $n(T)=1+\sum_{i=1}^{d}\left(2 k_{i}+1\right)$ and size $m$ there are four types of minimal dominating sets. The set which contains the center vertex and all pendent vertices, the set which contains the center vertex and all vertices adjacent to the pendent vertices, the set which contains the roots of $\beta_{1}, \beta_{2}, \ldots, \beta_{d}$ and all pendent vertices, and the set which contains the roots of $\beta_{1}, \beta_{2}, \ldots, \beta_{d}$ and all vertices adjacent to the pendent vertices. Hence, $d_{d}(v)=2$ for all $v \in V(T)$. So by using the definition of domination Zagreb indices we get the result.

\section{Definition 3.4.}

Let $G_{1}$ and $G_{2}$ be any two graphs. The Cartesian product $G_{1} \times G_{2}$ is defined as [6] the graph has vertex set $\left(V\left(G_{1}\right) \times V\left(G_{2}\right)\right)$ such that any two vertices $u=\left(u_{1}, u_{2}\right)$ and $v=\left(v_{1}, v_{2}\right)$ are adjacent if and only if either $\left(\left[u_{1}=v_{1}\right.\right.$ and $\left.\left.\left\{u_{2}, v_{2}\right\} \in E\left(G_{2}\right)\right]\right)$ or $\left(\left[u_{2}=v_{2}\right.\right.$ and $\left.\left.\left\{u_{1}, v_{1}\right\} \in E\left(G_{1}\right)\right]\right)$.

\section{Definition 3.5.}


Book graph $B_{r}$ is a Cartesian product of a star and single edge $S_{r+1} \times P_{2}$. The generalization of the book graph to $n$ "stacked" is the $(r, s)$-Stacked book graph [13].

\section{Lemma 3.4.}

If $G \cong B_{r}$, then $T_{m}(G)=2^{r}+3$. Further, for any vertex $v \in V\left(B_{r}\right)$

$d_{d}(v)= \begin{cases}3, & \text { if } v \text { is the center vertex; } \\ 2^{r-1}+1, & \text { otherwise }\end{cases}$

Proof. Let $u v$ be the center edge in book graph such that $\{u, v\}$ is the set of center vertices. Let $\left\{v_{1}, v_{2}, \ldots, v_{r}\right\}$ be the set of all vertices which are adjacent with the center vertex $v$. Similarly $\left\{u_{1}, u_{2}, \ldots, u_{r}\right\}$ be the set of all vertices which are adjacent with the center vertex $u$. There are four types of minimal dominating sets. First type is $\{u, v\}$. Second type is $\left\{v, u_{1}, u_{2}, \ldots, u_{r}\right\}$ and $\left\{u, v_{1}, v_{2}, \ldots, v_{r}\right\}$. Third type is $\left\{u, u_{1}, u_{2}, \ldots, u_{r}\right\}$ and $\left\{v, v_{1}, v_{2}, \ldots, v_{r}\right\}$. Fourth type is only those minimal dominating sets which are formed by taking one vertex from each section other than $u$ and $v$. So there are $2^{r}-2$ minimal dominating sets of fourth type. Hence, $T_{m}\left(B_{r}\right)=2^{r}+3$ and for all $v \in V\left(B_{r}\right)$ we get

$d_{d}(v)= \begin{cases}3, & \text { if } \mathrm{v} \text { is the center vertex } \\ 2^{r-1}+1, & \text { otherwise }\end{cases}$

\section{Theorem 3.3.}

Let $G$ be a book graph $B_{r}$ where $r \geq 3$. Then

$$
\begin{gathered}
D M_{1}\left(B_{r}\right)=2 r\left(2^{r-1}+1\right)^{2}+18, \\
D M_{2}\left(B_{r}\right)=r\left(2^{r-1}+1\right)\left[2^{r-1}+7\right]+9, \\
D M_{1}^{*}\left(B_{r}\right)=2^{r+1} r+2 r\left(4+2^{r-1}\right)+6 .
\end{gathered}
$$

Proof.

$$
\begin{aligned}
D M_{1}\left(B_{r}\right) & =\sum_{w \in V\left(B_{r}\right)} d_{d}^{2}(w)=\sum_{w \in V\left(B_{r}-\{u, v\}\right)}\left(2^{r-1}+1\right)^{2}+\sum_{w \in\{u, v\}} 3^{2} \\
& =2 r\left(2^{r-1}+1\right)^{2}+18 .
\end{aligned}
$$

There are three type of edges in the book graph.

Let $E_{1}$ denote the set of $r$ edges $\left(u_{i} v_{i}\right)$ with initial and terminal vertices of the same domination degree $2^{r-1}+1, E_{2}$ denote the set containing only one edge $(u v)$ with initial and terminal vertices of the same domination degree 3 , and $E_{3}$ denote the set of $2 r$ edges of initial vertices of the domination degree 3 and terminal vertices of domination degree $2^{r-1}+1$. Hence, 


$$
\begin{gathered}
D M_{2}\left(B_{r}\right)=\sum_{u v \in E\left(B_{r}\right)} d_{d}(u) d_{d}(v) \\
=\sum_{u v \in E_{1}}\left(2^{r-1}+1\right)^{2}+\sum_{u n \in E_{2}} 9+\sum_{u v \in E_{3}} 3\left(2^{r-1}+1\right) \\
=r\left(2^{r-1}+1\right)\left[2^{r-1}+7\right]+9 \\
D M_{1}^{*}\left(B_{r}\right)=\sum_{u v \in E\left(B_{r}\right)}\left[d_{d}(u)+d_{d}(v)\right] \\
=\sum_{u v \in E_{1}}\left[\left(2^{r-1}+1\right)+\left(2^{r-1}+1\right)\right]+\sum_{u v \in E_{2}}[3+3]+\sum_{u v \in E_{3}}\left[3+2^{r-1}+1\right] \\
=2^{r+1}+2 r\left(4+2^{r-1}\right)+6 .
\end{gathered}
$$

\section{Lemma 3.5.}

Let $G \cong K_{n_{1}, n_{2}, \ldots, n_{k}}$. Then

$$
T_{m}(G)=\sum_{i=2}^{k} n_{1} n_{i}+\sum_{i=3}^{k} n_{2} n_{i}+\ldots+n_{k-1} n_{k}+k .
$$

\section{Theorem 3.4.}

If $G \cong K_{n_{1}, n_{2}, \ldots, n_{k}}$, then

$$
\begin{gathered}
D M_{1}(G)=M_{1}(G)+4\left(T_{m}(G)-k\right)+\sum_{i=1}^{k} n_{i}, \\
D M_{2}(G)=M_{2}(G)+M_{1}(G)+T_{m}(G)-k, \\
D M_{1}^{*}(G)=M_{1}(G)+2\left(T_{m}(G)-k\right) .
\end{gathered}
$$

Proof. Suppose $G \cong K_{n_{1}, n_{2}, \ldots, n_{k}}$, note that for any vertex $v \in G$ we have $d_{d}(v)=d(v)+1$, and $|E(G)|=$ $T_{m}(G)-k$. So, by the definition of domination Zagreb indices we get the result.

\section{Lemma 3.6.}

For any connected graph $G$ with $n_{1}$ vertices and $m_{1}$ edges. Let $H \cong G \circ K_{n_{2}}$, where $K_{n_{2}}$ is the complete graph of $n_{2}$ vertices and $m_{2}$ edges. There are $\left(n_{2}+1\right)^{n_{1}}$ minimal domination sets in $H$, and $d_{d}(v)=\left(n_{2}+1\right)^{n_{1}-1}$.

\section{Theorem 3.5.}

For any connected graph $G$ of $n_{1}$ vertices and $m_{1}$ edges, we have

$$
\begin{gathered}
D M_{1}\left(G \circ K_{n_{2}}\right)=\left(n_{1}+n_{1} n_{2}\right)\left(n_{2}+1\right)^{2\left(n_{1}-1\right)}, \\
D M_{2}\left(G \circ K_{n_{2}}\right)=\left(n_{2}+1\right)^{2\left(n_{1}-1\right)}\left[2 m_{1}+n_{2}\left(n_{2}+2 n_{1}-1\right)\right], \\
D M_{1}^{*}\left(G \circ K_{n_{2}}\right)=4\left(n_{2}+1\right)^{n_{1}-1}\left[2 m_{1}+n_{2}\left(n_{2}+2 n_{1}-1\right)\right] .
\end{gathered}
$$


Proof. Note that $\left|V\left(G \circ K_{n_{2}}\right)\right|=n_{1}+n_{1} n_{2}$. Hence, by the definition of first domination Zagreb indices and Lemma 3.6, we get

$$
D M_{1}\left(G \circ K_{n_{2}}\right)=\left(n_{1}+n_{1} n_{2}\right)\left(n_{2}+1\right)^{2\left(n_{1}-1\right)} .
$$

There are three types of edges in $G \circ K_{n_{2}}$. All edges of $G$, all edges of $K_{n_{2}}$ and let $E_{1}$ denote the set of all edges that connect vertex from $G$ and vertex from $K_{n_{2}}$. So, we have

$$
\begin{aligned}
D M_{2}\left(G \circ K_{n_{2}}\right) & =\sum_{u v \in E\left(G o K_{n_{2}}\right)} d_{d}(u) d_{d}(v) \\
& =\sum_{u v \in E(G)} d_{d}(u) d_{d}(v)+\sum_{u v \in E\left(K_{n_{2}}\right)} d_{d}(u) d_{d}(v)+\sum_{u v \in E_{1}} d_{d}(u) d_{d}(v) \\
& =m_{1}\left(n_{2}+1\right)^{2\left(n_{1}-1\right)}+\left(n_{2}+1\right)^{2\left(n_{1}-1\right)}\left|E\left(K_{n_{2}}\right)\right|+n_{1} n_{2}\left(n_{2}+1\right)^{2\left(n_{1}-1\right)} \\
& =\left(n_{2}+1\right)^{2 n_{1}-2}\left[m_{1}+\frac{n_{2}\left(n_{2}-1\right)}{2}+n_{1} n_{2}\right] \\
& =\left(n_{2}+1\right)^{2\left(n_{1}-1\right)}\left[2 m_{1}+n_{2}\left(n_{2}+2 n_{1}-1\right)\right], \\
D M_{1}^{*}\left(G \circ K_{n_{2}}\right)= & \sum_{u v \in E\left(G \circ K_{n_{2}}\right)}\left[d_{d}(u)+d_{d}(v)\right] \\
= & \sum_{u v \in E(G)} 2\left(n_{2}+1\right)^{n_{1}-1}+\sum_{u v \in E\left(K_{n_{2}}\right)} 2\left(n_{2}+1\right)^{n_{1}-1}+\sum_{u v \in E_{1}} 2\left(n_{2}+1\right)^{n_{1}-1} \\
= & 4\left(n_{2}+1\right)^{n_{1}-1}\left[2 m_{1}+n_{2}\left(n_{2}+2 n_{1}-1\right)\right] .
\end{aligned}
$$

\section{Lemma 3.7.}

Let $H \cong G \circ \overline{K_{n_{2}}}$, where $G$ be any connected graph of order $n_{1}$. Then

$$
T_{m}(H)=\sum_{i=0}^{n_{1}}\left(\begin{array}{c}
n_{1} \\
i
\end{array}\right) .
$$

\section{Theorem 3.6.}

If $G$ be a graph of order $n_{1}$ and size $m_{1}$. Let $H \cong G \circ \overline{K_{n_{2}}}$ then

$$
\begin{aligned}
& D M_{1}(H)=\left(T_{m}(H)-2^{n_{1}-1}\right)^{2}\left(n_{1}+n_{1} n_{2}\right), \\
& D M_{2}(H)=\left(T_{m}(H)-2^{n_{1}-1}\right)^{2}\left(m_{1}+n_{1} n_{2}\right), \\
& D M^{*_{1}}(H)=\left(2 T_{m}(H)-2^{n_{1}}\right)\left(m_{1}+n_{1} n_{2}\right) .
\end{aligned}
$$

Proof. For any vertex $v \in V(H)$, it is not easy to see that $H \cong G \circ \overline{K_{n_{2}}}$ is domination regular graph. Every $v \in V(H)$ is contained in every minimal dominating sets of $H$ except 
$\left(\begin{array}{c}n_{1}-1 \\ 0\end{array}\right)+\left(\begin{array}{c}n_{1}-1 \\ 1\end{array}\right)+\ldots+\left(\begin{array}{c}n_{1}-1 \\ n_{1}-2\end{array}\right)+\left(\begin{array}{c}n_{1}-1 \\ n_{1}-1\end{array}\right)=2^{n_{1}-1}$ minimal dominating sets.

Hence, $d_{d H}(v)=T_{m}(H)-2^{n_{1}-1}$ and

$$
\begin{aligned}
& D M_{1}(H)=\left(T_{m}(H)-2^{n_{1}-1}\right)^{2}\left(n_{1}+n_{1} n_{2}\right), \\
& D M_{2}(H)=\left(T_{m}(H)-2^{n_{1}-1}\right)^{2}\left(m_{1}+n_{1} n_{2}\right), \\
& D M^{*_{1}}(H)=\left(2 T_{m}(H)-2^{n_{1}}\right)\left(m_{1}+n_{1} n_{2}\right) .
\end{aligned}
$$

A join of two graphs $G_{1}$ and $G_{2}$ is denoted by $G_{1}+G_{2}$, with disjoint vertex sets $V_{1}$ and $V_{2}$ is the graph on the vertex set $V_{1} \cup V_{2}$ and the edge set $E_{1} \cup E_{2} \cup\left\{u_{1} u_{2}: u_{1} \in V_{1}, u_{2} \in V_{2}\right\}$ [1].

\section{Lemma 3.8.}

Let $G_{1}$ and $G_{2}$ be any non complete graphs of $n_{1}, n_{2}$ vertices respectively. Then

$T_{m}\left(G_{1}+G_{2}\right)=T_{m}\left(G_{1}\right)+T_{m}\left(G_{2}\right)+n_{1} n_{2}$, and

$d_{d G_{1}+G_{2}}(v)= \begin{cases}d_{d G_{1}}(v)+n_{2}, & \text { if } v \in V\left(G_{1}\right) ; \\ d_{d G_{2}}(v)+n_{1}, & \text { if } v \in V\left(G_{2}\right) .\end{cases}$

Proof. There are three types of minimal dominating sets in $G_{1}+G_{2}$ graph:

The minimal-dominating sets of $G_{1}$, all the minimal dominating sets of $G_{2}$ and the sets of size two of all minimal dominating sets containing one vertex from $G_{1}$ and another vertex from $G_{2}$.

Hence, $T_{m}\left(G_{1}+G_{2}\right)=T_{m}\left(G_{1}\right)+T_{m}\left(G_{2}\right)+n_{1} n_{2}$, and

$d_{d G_{1}+G_{2}}(v)= \begin{cases}d_{d G_{1}}(v)+n_{2}, & \text { if } v \in V\left(G_{1}\right) ; \\ d_{d G_{2}}(v)+n_{1}, & \text { if } v \in V\left(G_{2}\right) .\end{cases}$

\section{Theorem 3.7.}

Let $G_{1}$ and $G_{2}$ be any non complete graphs having $n_{1}, n_{2}$ vertices and $m_{1}, m_{2}$ edges respectively. Then

$$
\begin{aligned}
D M_{1}\left(G_{1}+G_{2}\right) & =D M_{1}\left(G_{1}\right)+D M_{2}\left(G_{2}\right)+2 n_{2} \rho\left(G_{1}\right) \\
& +2 n_{1} \rho\left(G_{2}\right)+n_{1}\left(n_{2}^{2}+n_{2} n_{1}\right)
\end{aligned}
$$

$$
\begin{aligned}
D M_{2}\left(G_{1}+G_{2}\right) & =D M_{2}\left(G_{1}\right)\left(1+n_{2}\right)+m_{1} n_{2}^{2}+D M_{2}\left(G_{2}\right)\left(1+n_{1}\right)+m_{2} n_{1}^{2} \\
& +\left[n_{1} n_{2}+\rho\left(G_{1}\right)\right]\left[n_{1} n_{2}+\rho\left(G_{2}\right)\right]
\end{aligned}
$$


(3)

$$
\begin{aligned}
D M_{1}^{*}\left(G_{1}+G_{2}\right) & =D M_{1}^{*}\left(G_{1}\right)+D M_{1}^{*}\left(G_{2}\right)+2 m_{1} n_{2}+2 n_{1} m_{2} \\
& +\left(\rho\left(G_{2}\right)+n_{2}\left(n_{1}+1\right)\right)\left(\rho\left(G_{1}\right)+n_{1} n_{2}\right) .
\end{aligned}
$$

Proof.

$$
\begin{aligned}
D M_{1}\left(G_{1}+G_{2}\right) & =\sum_{v \in V\left(G_{1}+G_{2}\right)} d_{d G_{1}+G_{2}}^{2}(v) \\
& =\sum_{v \in V\left(G_{1}\right)}\left(d_{d G_{1}}(v)+n_{2}\right)^{2}+\sum_{v \in V\left(G_{2}\right)}\left(d_{d G_{2}}(v)+n_{1}\right)^{2} \\
& =\sum_{v \in V\left(G_{1}\right)} d_{d G_{1}}(v)^{2}+2 n_{2} \sum_{v \in V\left(G_{1}\right)} d_{d G_{1}}(v)+n_{2}^{2} \sum_{v \in V\left(G_{1}\right)} 1 \\
& +\sum_{v \in V\left(G_{2}\right)} d_{d G_{2}}(v)^{2}+2 n_{1} \sum_{v \in V\left(G_{2}\right)} d_{d G_{2}}(v)+n_{1}^{2} \sum_{v \in V\left(G_{1}\right)} 1 \\
& =D M_{1}\left(G_{1}\right)+D M_{2}\left(G_{2}\right)+2 n_{2} \sum_{v \in V\left(G_{1}\right)} d_{d G_{1}}(v) \\
& +2 n_{1} \sum_{v \in V\left(G_{2}\right)} d_{d G_{2}}(v)+n_{1}\left(n_{2}^{2}+n_{2} n_{1}\right) \\
& =D M_{1}\left(G_{1}\right)+D M_{2}\left(G_{2}\right)+2 n_{2} \rho\left(G_{1}\right) \\
& +2 n_{1} \rho\left(G_{2}\right)+n_{1}\left(n_{2}^{2}+n_{2} n_{1}\right) .
\end{aligned}
$$

And,

$$
\begin{aligned}
D M_{2}\left(G_{1}+G_{2}\right) & =\sum_{u v \in E\left(G_{1}+G_{2}\right)} d_{d G_{1}+G_{2}}(u) d_{d G_{1}+G_{2}}(v) \\
& =\sum_{u v \in E\left(G_{1}\right)} d_{d G_{1}+G_{2}}(u) d_{d G_{1}+G_{2}}(v)+\sum_{u v \in E\left(G_{2}\right)} d_{d G_{1}+G_{2}}(u) d_{d G_{1}+G_{2}}(v) \\
& +\sum_{u \in V\left(G_{1}\right), v \in V\left(G_{2}\right)} d_{d G_{1}+G_{2}}(u) d_{d G_{1}+G_{2}}(v)
\end{aligned}
$$

We will find every part independently

(1)

$$
\begin{aligned}
\sum_{u v \in E\left(G_{1}\right)} d_{d G_{1}+G_{2}}(u) d_{d G_{1}+G_{2}}(v) & =\sum_{u v \in E\left(G_{1}\right)}\left(d_{d G_{1}}(u)+n_{2}\right)\left(d_{d G_{1}}(v)+n_{2}\right) \\
& =D M_{2}\left(G_{1}\right)\left(1+n_{2}\right)+m_{1} n_{2}^{2}
\end{aligned}
$$


(2)

$$
\begin{aligned}
\sum_{u v \in E\left(G_{2}\right)} d_{d G_{1}+G_{2}}(u) d_{d G_{1}+G_{2}}(v) & =\sum_{u v \in E\left(G_{2}\right)}\left(d_{d G_{2}}(u)+n_{1}\right)\left(d_{d G_{2}}(v)+n_{1}\right) \\
& =D M_{2}\left(G_{2}\right)\left(1+n_{1}\right)+m_{2} n_{1}^{2}
\end{aligned}
$$

$$
\begin{aligned}
\sum_{u \in V\left(G_{1}\right), v \in V\left(G_{2}\right)} d_{d G_{1}+G_{2}}(u) d_{d G_{1}+G_{2}}(v) & =\left(d_{d G_{1}}\left(u_{1}\right)+n_{2}\right)\left(d_{d G_{2}}\left(v_{1}\right)+n_{1}\right)+\ldots \\
& +\left(d_{d G_{1}}\left(u_{1}\right)+n_{2}\right)\left(d_{d G_{2}}\left(v_{n_{2}}\right)+n_{1}\right) \\
& +\left(d_{d G_{1}}\left(u_{2}\right)+n_{2}\right)\left(d_{d G_{2}}\left(v_{1}\right)+n_{1}\right)+\ldots \\
& +\left(d_{d G_{1}}\left(u_{2}\right)+n_{2}\right)\left(d_{d G_{2}}\left(v_{n_{2}}\right)+n_{1}\right) \\
& +\ldots \\
& +\left(d_{d G_{1}}\left(u_{n_{1}}\right)+n_{2}\right)\left(d_{d G_{2}}\left(v_{1}\right)+n_{1}\right)+\ldots \\
& +\left(d_{d G_{1}}\left(u_{n_{1}}\right)+n_{2}\right)\left(d_{d G_{2}}\left(v_{n_{2}}\right)+n_{1}\right) \\
& =\left(d_{d G_{1}}\left(u_{1}\right)+n_{2}\right)\left[\sum_{v \in V\left(G_{2}\right)}\left(d_{d G_{2}}(v)+n_{1}\right)\right]+\ldots \\
& +\left(d_{d G_{1}}\left(u_{n_{1}}\right)+n_{2}\right)\left[\sum_{v \in V\left(G_{2}\right)}\left(d_{d G_{2}}(v)+n_{1}\right)\right] \\
& =\left[\sum_{u \in V\left(G_{1}\right)}\left(d_{d G_{1}}(u)+n_{2}\right)\right]\left[\sum_{v \in V\left(G_{2}\right)}\left(d_{d G_{2}}(v)+n_{1}\right)\right] \\
& =\left[n_{1} n_{2}+\sum_{u \in V\left(G_{1}\right)} d_{d G_{1}}(u)\right]\left[n_{1} n_{2}+\sum_{v \in V\left(G_{2}\right)} d_{d G_{2}}(v)\right] \\
& =\left[n_{1} n_{2}+\rho\left(G_{1}\right)\right]\left[n_{1} n_{2}+\rho\left(G_{2}\right)\right]
\end{aligned}
$$

Hence,

$$
\begin{aligned}
D M_{2}\left(G_{1}+G_{2}\right) & =D M_{2}\left(G_{1}\right)\left(1+n_{2}\right)+m_{1} n_{2}^{2}+D M_{2}\left(G_{2}\right)\left(1+n_{1}\right)+m_{2} n_{1}^{2} \\
& +\left[n_{1} n_{2}+\rho\left(G_{1}\right)\right]\left[n_{1} n_{2}+\rho\left(G_{2}\right)\right] .
\end{aligned}
$$

And,

$$
\begin{aligned}
D M_{1}^{*}\left(G_{1}+G_{2}\right) & =\sum_{u v \in E\left(G_{1}+G_{2}\right)}\left[d_{d G_{1}+G_{2}}(u)+d_{d G_{1}+G_{2}}(v)\right] \\
& =\sum_{u v \in E\left(G_{1}\right)}\left[d_{d G_{1}+G_{2}}(u)+d_{d G_{1}+G_{2}}(v)\right]+\sum_{u v \in E\left(G_{2}\right)}\left[d_{d G_{1}+G_{2}}(u)+d_{d G_{1}+G_{2}}(v)\right] \\
& +\sum_{u \in V\left(G_{1}\right), v \in V\left(G_{2}\right)}\left[d_{d G_{1}+G_{2}}(u)+d_{d G_{1}+G_{2}}(v)\right] .
\end{aligned}
$$


We will find every part independently

(1)

$$
\begin{aligned}
\sum_{u v \in E\left(G_{1}\right)}\left[d_{d G_{1}+G_{2}}(u)+d_{d G_{1}+G_{2}}(v)\right] & =\sum_{u v \in E\left(G_{1}\right)}\left(d_{d G_{1}}(u)+n_{2}\right)+\left(d_{d G_{1}}(v)+n_{2}\right) \\
& =D M_{1}^{*}\left(G_{1}\right)+2 m_{1} n_{2}
\end{aligned}
$$

$$
\begin{aligned}
\sum_{u v \in E\left(G_{2}\right)}\left[d_{d G_{1}+G_{2}}(u)+d_{d G_{1}+G_{2}}(v)\right] & =\sum_{u v \in E\left(G_{2}\right)}\left(d_{d G_{2}}(u)+n_{1}\right)+\left(d_{d G_{2}}(v)+n_{1}\right) \\
& =D M_{1}^{*}\left(G_{2}\right)+2 n_{1} m_{2},
\end{aligned}
$$

(3)

$$
\begin{aligned}
\sum_{u \in V\left(G_{1}\right), v \in V\left(G_{2}\right)}\left[d_{d G_{1}+G_{2}}(u)+d_{d G_{1}+G_{2}}(v)\right] & =\left(d_{d G_{1}}\left(u_{1}\right)+n_{2}\right)+\left(d_{d G_{2}}\left(v_{1}\right)+n_{1}\right)+\ldots \\
& +\left(d_{d G_{1}}\left(u_{1}\right)+n_{2}\right)+\left(d_{d G_{2}}\left(v_{n_{2}}\right)+n_{1}\right) \\
& +\left(d_{d G_{1}}\left(u_{2}\right)+n_{2}\right)+\left(d_{d G_{2}}\left(v_{1}\right)+n_{1}\right)+\ldots \\
& +\left(d_{d G_{1}}\left(u_{2}\right)+n_{2}\right)+\left(d_{d G_{2}}\left(v_{n_{2}}\right)+n_{1}\right) \\
& +\ldots \\
& +\left(d_{d G_{1}}\left(u_{n_{1}}\right)+n_{2}\right)+\left(d_{d G_{2}}\left(v_{1}\right)+n_{1}\right)+\ldots \\
& +\left(d_{d G_{1}}\left(u_{n_{1}}\right)+n_{2}\right)+\left(d_{d G_{2}}\left(v_{n_{2}}\right)+n_{1}\right) \\
& =\left(d_{d G_{1}}\left(u_{1}\right)+n_{2}\right)\left[\sum_{v \in V\left(G_{2}\right)}\left(d_{d G_{2}}(v)+n_{1}\right)+n_{2}\right]+\ldots \\
& +\left(d_{d G_{1}}\left(u_{n_{1}}\right)+n_{2}\right)\left[\sum_{v \in V\left(G_{2}\right)}\left(d_{d G_{2}}(v)+n_{1}\right)+n_{2}\right] \\
& \left.=\left[\sum_{v \in V\left(G_{2}\right)}\left(d_{d G_{2}}(v)\right)+n_{2}\left(n_{1}+1\right)\right]\left[\sum_{u \in V\left(G_{1}\right)}\left(d_{d G_{1}}(u)\right)+n_{1} n_{2}\right)\right] \\
& =\left(\rho\left(G_{2}\right)+n_{2}\left(n_{1}+1\right)\right)\left(\rho\left(G_{1}\right)+n_{1} n_{2}\right) .
\end{aligned}
$$

Hence,

$$
\begin{aligned}
D M_{1}^{*}\left(G_{1}+G_{2}\right) & =D M_{1}^{*}\left(G_{1}\right)+D M_{1}^{*}\left(G_{2}\right)+2 m_{1} n_{2}+2 n_{1} m_{2} \\
& +\left(\rho\left(G_{2}\right)+n_{2}\left(n_{1}+1\right)\right)\left(\rho\left(G_{1}\right)+n_{1} n_{2}\right) .
\end{aligned}
$$




\section{Corollary 3.2.}

$$
\begin{aligned}
D M_{1}\left(G_{1}+G_{2}\right) & \leq D M_{1}\left(G_{1}\right)+D M_{2}\left(G_{2}\right)+2 n_{1} \Gamma\left(G_{1}\right) T_{m}\left(G_{1}\right) \\
& +2 n_{1} \Gamma\left(G_{2}\right) T_{m}\left(G_{2}\right)+n_{1}\left(n_{2}^{2}+n_{2} n_{1}\right) .
\end{aligned}
$$

\section{Corollary 3.3.}

$$
\begin{aligned}
D M_{2}\left(G_{1}+G_{2}\right) & \leq D M_{2}\left(G_{1}\right)\left(1+n_{2}\right)+m_{1} n_{2}^{2}+D M_{2}\left(G_{2}\right)\left(1+n_{1}\right)+m_{2} n_{1}^{2} \\
& +\left[n_{1} n_{2}+\Gamma\left(G_{1}\right) T_{m}\left(G_{1}\right)\right]\left[n_{1} n_{2}+\Gamma\left(G_{2}\right) T_{m}\left(G_{2}\right)\right] .
\end{aligned}
$$

\section{Corollary 3.4.}

$$
\begin{aligned}
D M_{1}^{*}\left(G_{1}+G_{2}\right) & \leq D M_{1}^{*}\left(G_{1}\right)+D M_{1}^{*}\left(G_{2}\right)+2 m_{1} n_{2}+2 n_{1} m_{2} \\
& +\left(\Gamma\left(G_{2}\right) T_{m}\left(G_{2}\right)+n_{2}\left(n_{1}+1\right)\right)\left(\Gamma\left(G_{1}\right) T_{m}\left(G_{1}\right)+n_{1} n_{2}\right) .
\end{aligned}
$$

\section{Some bounds of Domination Zagreb indices}

\section{Theorem 4.1.}

Let $G$ be a graph of order $n$. Then

$D M_{1}(G) \geq \frac{1}{n}(\rho(G))^{2}$. Equality hold if and only if $G$ is one-domination regular graph.

Proof. We have $D M_{1}(G)=\sum_{v \in V(G)} d_{d}^{2}(v)=d_{d}^{2}\left(v_{1}\right)+d_{d}^{2}\left(v_{2}\right)+\ldots+d_{d}^{2}\left(v_{n}\right)$

We use Cauchy-schwartz inequality on vectors $\left(d_{d}\left(v_{1}\right), d_{d}\left(v_{2}\right), \ldots, d_{d}\left(v_{n}\right)\right)$ and $(1,1, \ldots, 1)$ to get

$$
\begin{aligned}
D M_{1}(G) . n & =\left(d_{d}^{2}\left(v_{1}\right), d_{d}^{2}\left(v_{2}\right), \ldots, d_{d}^{2}\left(v_{n}\right)\right)\left(1^{2}, 1^{2}, \ldots, 1^{2}\right) \\
& \geq\left(d_{d}\left(v_{1}\right) \cdot 1+d_{d}\left(v_{2}\right) \cdot 1+\ldots+d_{d}\left(v_{n}\right) \cdot 1\right)^{2} \\
& =\left(\sum_{i=1}^{n} d_{d}\left(v_{i}\right)\right)^{2} \\
& =(\rho(G))^{2} .
\end{aligned}
$$

To prove the equality, suppose $G$ is one domination regular graph $\Rightarrow d d\left(v_{i}\right)=1$ for all $1 \leq i \leq n$. And $D M_{1}(G)=n$. Conversely if $D M_{1}(G)=\frac{1}{n}\left(\sum_{i=1}^{n} d_{d}\left(v_{i}\right)\right)^{2} \Rightarrow D M_{1}(G) \cdot n=\left(\sum_{i=1}^{n} d\left(v_{i}\right)\right)^{2}$, hence $G$ is one domination regular graph.

\section{Theorem 4.2.}

Let $G$ be a graph with $n$ vertices. Then

$$
D M_{1}(G) \leq\left(\sum_{v \in V(G)} \sqrt{d_{d}(v)}\right)^{2}
$$


Proof. $D M_{1}(G)=\sum_{v \in V(G)} d_{d}^{2}(v)=d_{d}^{2}\left(v_{1}\right)+d_{d}^{2}\left(v_{2}\right)+\ldots+d_{d}^{2}\left(v_{n}\right)$

As $d_{d}\left(v_{1}\right), d_{d}\left(v_{2}\right), \ldots, d_{d}\left(v_{n}\right)$ is positive integers so, we get

$$
D M_{1}(G) \leq\left(\sum_{v \in V(G)} \sqrt{d_{d}(v)}\right)^{2}
$$

\section{Proposition 4.1.}

If $G$ be any graph such that $|V(G)|=n$, then

$$
\begin{gathered}
n \leq D M_{1}(G) \leq n\left(T_{m}(G)\right)^{2}, \\
\rho(G)+n \leq D M_{1}^{*}(G) \leq n T_{m}(G)+\rho(G) .
\end{gathered}
$$

Proof. see Observation 2.1.

\section{Theorem 4.3.}

Let $G$ be a graph such that $G ¥ K_{2}$. Then $D M_{2}(G) \geq \gamma(G) T_{m}(G)$

Equality hold if and only if $G \cong P_{3}$.

Proof. Note that $d_{d}(v) \geq 1$, so, $\sum_{u v \in E(G)} d_{d}(u) d_{d}(v) \geq \sum_{u \in V(G)} d_{d}(u)$. From Observation 2.3, we get $D M_{2}(G) \geq \gamma(G) T_{m}(G)$.

\section{Theorem 4.4.}

Suppose $G$ is a connected simple graph. Then

$D M_{2}(G) \leq \Gamma(G)\left(T_{m}(G)\right)^{2}$.

Proof. We have $d_{d}(v) \leq T_{m}(G)$, so $\sum_{u v \in E(G)} d_{d}(u) d_{d}(v) \leq T_{m}(G) \sum_{u \in V(G)} d d(u)$.

By Observation 2.3, $D M_{2}(G) \leq \Gamma(G)\left(T_{m}(G)\right)^{2}$.

Finally, we can generalize the definition of the domination degree of the vertex by using any subset of vertices with property $P$ like an independent set, independent dominating set, total dominating set, hup set, edge dominating set, different distance set,... so on.

\section{Definition 4.1.}

Let $G=(V, E)$ be a graph and let $S$ be any subset of vertices with property $P$. Then for any vertex $v$, the $P$ set degree of the vertex $v$ denoted by

$$
d_{P}(v)=\mid\{S \subseteq V(G): S \text { has property } P \text { and } v \in S\} \mid
$$


And we can define the Zagreb and Forgotten indices as following

$$
\begin{gathered}
P M_{1}(G)=\sum_{v \in V(G)} d_{P}^{2}(v), \\
P M_{1}^{*}(G)=\sum_{u v \in E(G)} d_{P}(u)+d_{P}(v), \\
P M_{2}(G)=\sum_{u v \in E(G)} d_{P}(u) d_{P}(v), \\
P F(G)=\sum_{v \in V(G)} d_{P}^{3}(v), \\
P F^{*}(G)=\sum_{u v \in E(G)} d_{P}^{2}(u)+d_{P}^{2}(v) .
\end{gathered}
$$

\section{CONClusion}

In this research work, we define new topological indices based on the minimal dominating sets. The authors are working now in some of the other types of topological indices by replacing the standers degree of the vertex by the domination degree of the vertex.

Finally we have the following open problems for research:

(1) Is there any relation between the normal degree and domination degree of the graph.

(2) What is the necessary and sufficient conditions in a graph to become domination regular graph.

(3) What is the natural relation between the domination indices.

Conflicts of Interest: The author(s) declare that there are no conflicts of interest regarding the publication of this paper.

\section{REFERENCES}

[1] A.R. Ashrafi, T. Doslic, A. Hamzeha, The Zagreb coindices of graph operations, Discrete Appl. Math. 158 (2010), 15711578.

[2] T. Al-Fozan, P. Manuel, I. Rajasingh, R. Sundara Rajan, Computing Szeged Index of Certain Nanosheets Using Partition Technique, MATCH Commun. Math. Comput. Chem. 72 (2014), 339-353.

[3] M. Azari, A. Iranmanesh, Harary Index of Some Nano-Structures, MATCH Commun. Math. Comput. Chem. 71 (2014), $373-382$.

[4] B. Basavanagoud, On minimal and vertex minimal dominating graph, J. Inform. Math. Sci. 1 (2009), $139-146$.

[5] D. Dimitrov, On structural properties of trees with minimal atom-bond connectivity index II: Bounds on B 1 - and B 2 -branches, Discrete Appl. Math. 204 (2016), 90-116.

[6] T. Došlić, M. Hosseinzadeh, Eccentric connectivity polynomial of some graph operations, Utilitas Math. 84 (2011), $297-309$.

[7] F.V. Fomin, F. Grandoni, A.V. Pyatkin, A.A. Stepanov. Combinatorial bounds via measure and conquer: bounding minimal dominating sets and applications. ACM Trans. Algorithms 5(1) (2008), 9.

[8] B. Furtula, I. Gutman, and M. Dehmer, On structure-sensitivity of degree-based topological indices, Appl. Math. Comput. 219 (2013), 8973-8978.

[9] I. Gutman, Degree-based topological indices, Croat. Chem. Acta, 86 (2013), 351—361. 
[10] I. Gutman, K.C. Das, The first zagreb index 30 years after, MATCH Commun. Math. Comput. Chem. 50 (2004) 83 - 92.

[11] I. Gutman, B.Ruščić, N.Trinajstić, C.F. Wilcox, Graph theory and molecular orbitals. XII. Acyclic polyenes, J. Chem. Phys. 62 (1975), 3399-3405.

[12] I. Gutman, N. Trinajstić, Graph theory and molecular orbitals. total $\pi$-electron energy of alternant hydrocarbons, Chem. Phys. Lett. 17 (1972), 535-538.

[13] F. Harary, Graph Theory, Narosa Publishing House, New Delhi, 2001.

[14] T.W. Haynes, S.T. Hedetniemi, P.J.Slater, Fundamentals of Domination in Graphs, Marcel Dekker, New York. (1998).

[15] T.W. Haynes, S.T. Hedetniemi, P.J. Slater, (eds.) Domination in Graphs: Advanced Topics. Marcel Dekker, Inc. New York. (1998).

[16] Ivan Gutman, Kragujevac Trees and Their Energy, Appl. Math. Inform. Mech. 2 (2014), 71-79.

[17] O. Ivanciuc, T.S. Balaban, and A.T. Balaban, Reciprocal distance matrix, related local vertex invariants and topological indices, J. Math. Chem. 12 (1993), 309-318.

[18] F.C. Jear, R. Letourneur, M. Liedloff, On the number of minimal dominating sets on some graph classes, Theor. Comput. Sci. 562 (2015), 634-642.

[19] B.N. Kavitha, I.P. Kelkar, K.R. Rajanna, Perfect Domination in Book Graph and Stacked Book Graph, Int. J. Math. Trends Technol. 56 (2018), 511-514.

[20] M.F. Nadeem, S. Zafar, Z. Zahid, On certain topological indices of the line graph of subdivision graphs, Appl. Math. Comput. 271 (2015), 790-794.

[21] P.S. Ranjini, V. Lokesha, The Smarandache-Zagreb Indices on the Three Graph Operators, Int. J. Math. Comb. 3 (2010), $1-10$.

[22] V. Sharma, R. Goswami, A.K. Madan, Eccentric connectivity index: A novel highly discriminating topological descriptor for structure-property and structure-activity studies, J. Chem. Inf. Comput. Sci. 37 (1997), 273-282.

[23] M. Teresa de Bustos Muñoz, J.L.G. Guirao, J. Vigo-Aguiar, Decomposition of pseudo-radioactive chemical products with a mathematical approach, J. Math. Chem. 52 (4) (2014), 1059-1065.

[24] H. Wiener, Structural determination of the paraffin boiling points, J. Amer. Chem. Soc. 69 (1947), 17-20. 